\title{
Spermidine attenuates bleomycin-induced lung fibrosis by inducing autophagy and inhibiting endoplasmic reticulum stress (ERS)-induced cell death in mice
}

\author{
Ae Rin Baek', Jisu Hong ${ }^{1}$, Ki Sung Song ${ }^{1}$, An Soo Jang (1) ${ }^{1}$, Do Jin Kim', Su Sie Chin ${ }^{2}$ and Sung Woo Park (D)
}

\begin{abstract}
Spermidine is an endogenous biological polyamine that plays various longevity-extending roles and exerts antioxidative, antiaging, and cell growth-promoting effects. We previously reported that spermidine levels were significantly reduced in idiopathic pulmonary fibrosis (IPF) of the lung. The present study assessed the potential beneficial effects of spermidine on lung fibrosis and investigated the possible mechanism. Lung fibrosis was established in mice using bleomycin (BLM), and exogenous spermidine was administered daily by intraperitoneal injection (50 mg/kg in phosphate-buffered saline). BLM-induced alveolar epithelial cells showed significant increases in apoptosis and endoplasmic reticulum stress (ERS)-related mediators, and spermidine attenuated BLM-induced apoptosis and activation of the ERS-related pathway. Senescence-associated $\beta$-gal staining and decreased expression of p16 and p21 showed that spermidine ameliorated BLM-induced premature cellular senescence. In addition, spermidine enhanced beclin-1-dependent autophagy and autophagy modulators in IPF fibroblasts and BLM-induced mouse lungs, in which inflammation and collagen deposition were significantly decreased. This beneficial effect was related to the antiapoptotic downregulation of the ERS pathway, antisenescence effects, and autophagy activation. Our findings suggest that spermidine could be a therapeutic agent for IPF treatment.
\end{abstract}

\section{Introduction}

Idiopathic pulmonary fibrosis (IPF) is a progressive, devastating lung disease characterized by alveolar epithelial cell (AEC) injury and the subsequent proliferation of activated fibroblasts known as myofibroblasts. The accumulation of myofibroblasts is responsible for the excessive deposition of extracellular matrix (ECM), resulting in irreversible distortion of the lung parenchymal structure ${ }^{1,2}$. Although the exact mechanisms of the development of lung fibrosis remain unclear, repeated

\footnotetext{
Correspondence: Sung Woo Park (swpark@schmc.ac.kr)

${ }^{1}$ Division of Allergy and Respiratory Medicine, Department of Interna

Medicine, Soonchunhyang University Bucheon Hospital, 14584 Gyeonggi-Do, South Korea

²Department of Pathology, Soonchunhyang University Bucheon Hospital, 14584 Gyeonggi-Do, South Korea
}

These authors contributed equally: Ae Rin Baek, Jisu Hong injury and shortened survival of AECs have been recognized as initiating events ${ }^{3,4}$.

The precise mechanism of AEC damage remains unclear, but increased ERS or the accumulation of unfolded proteins in AECs have recently been implicated in IPF pathogenesis ${ }^{5,6}$. In addition, because the incidence of IPF increases with age, age-related mechanisms such as telomerase attrition and increased AEC senescence are also important drivers of the abnormal repair process during the initiation of fibrosis ${ }^{7,8}$.

Spermidine is a naturally occurring polyamine and is ubiquitous in living organisms as a polycation ${ }^{9,10}$. Polyamines are involved in various biological processes, including replication, transcription, translation, posttranslational modification, and membrane stability ${ }^{11}$, and they regulate cellular proliferation, differentiation, and

\section{(c) The Author(s) 2020}

(c) (i) Open Access This article is licensed under a Creative Commons Attribution 4.0 International License, which permits use, sharing, adaptation, distribution and reproduction cc in any medium or format, as long as you give appropriate credit to the original author(s) and the source, provide a link to the Creative Commons license, and indicate if changes were made. The images or other third party material in this article are included in the article's Creative Commons license, unless indicated otherwise in a credit line to the material. If material is not included in the article's Creative Commons license and your intended use is not permitted by statutory regulation or exceeds the permitted use, you will need to obtain permission directly from the copyright holder. To view a copy of this license, visit http://creativecommons.org/licenses/by/4.0/. 
apoptosis ${ }^{12,13}$. Previous studies have suggested that polyamines have multiple effects, including antioxidant and anti-inflammatory effects, in various animal disease models ${ }^{14}$. Eisenberg et al. ${ }^{15}$ reported that the exogenous administration of spermidine prolongs the life span of several organism models and significantly reduces agerelated oxidative protein damage in mice. In addition, natural endogenous spermidine has prominent cardioprotective and neuroprotective effects and stimulates anticancer immunosurveillance in animal models ${ }^{14}$.

We recently reported that spermidine levels were decreased in the IPF lung compared to healthy controls ${ }^{16}$. Although the beneficial effects of spermidine have been reported in various chronic inflammatory diseases, its role in and effects on lung fibrosis have not yet been elucidated. Given the antiaging and antioxidative activities of spermidine, we hypothesized that spermidine may have beneficial effects on lung fibrosis, particularly in regulating cellular senescence and ERS-induced cell death.

Hence, in this study, we examined whether spermidine exerted beneficial effects on experimental lung fibrosis. The mechanism of the beneficial effects of spermidine was determined using AEC culture models. The therapeutic implication of spermidine in lung fibrosis development was evaluated in a mouse model of bleomycin (BLM)induced lung fibrosis.

\section{Materials and methods \\ Reagents and antibodies}

The antibodies used were rabbit anti-p16 (\#ab51243; Abcam, Cambridge, UK), rabbit anti-p-Rb (\#8516; Cell Signaling Technology, Danvers, MA, USA), rabbit antiCHOP (\#5554; Cell Signaling Technology), rabbit antiGRP78/Bip (\#3177; Cell Signaling Technology), rabbit anti-ATF-6a (\#sc22799; Santa Cruz Biotechnology, Santa Cruz, CA, USA), rabbit anti-IRE-1 (\#ab37073; Abcam), rabbit anti-light chain (LC) 3B (\#3868S; Cell Signaling Technology), rabbit anti-autophagy related gene 7 (ATG7) (\#8558S; Cell Signaling Technology), rabbit antibeclin-1 (\#3738S; Cell Signaling Technology), rabbit antimTOR (\#2972; Cell Signaling Technology), rabbit antimTOR (phospho) (\#2971; Cell Signaling Technology), and mouse anti- $\beta$-actin (\#A5316; Sigma-Aldrich, St. Louis, MO, USA). Spermidine (\#S2626) and 4-PBA (\#P21005) were purchased from Sigma-Aldrich. An LDH assay kit (\#mk401; Takara) was used. The in situ cell death detection kit (\#11684795910; Roche Diagnostics, Basel, Switzerland), cellular senescence (SA- $\beta$-gal staining) assay kit (\#CBA-230; Cell Biolabs, San Diego, CA, USA), mouse interleukin-1 beta (IL-1 $\beta$ ) enzyme-linked immunosorbent assay (ELISA) kit (\#88701322; eBioscience, Inc., San Diego, CA, USA), mouse tumor necrosis factor-alpha (TNF- $\alpha$ ) ELISA kit (\#88732422; eBioscience, Inc.), and a mouse transforming growth factor-beta 1 (TGF- $\beta 1$ ) ELISA kit (\#DY1679; R\&D Systems, Minneapolis, MN, USA) were also purchased.

\section{Mouse AEC isolation and culture}

Primary mouse AECs were isolated from wild-type mice using a previously described protocol ${ }^{17}$ with minor modifications.

\section{Preparation of lung cell suspensions}

Crude cell suspensions were prepared from C57BL/6 mice. The lungs were perfused with $0.9 \% \mathrm{NaCl}$ using a $10-\mathrm{mL}$ syringe fitted with a 21-gauge needle (BD Pharmingen, San Diego CA, USA) through the right ventricle of the heart until they were visually free of blood. A 21gauge intravenous catheter was inserted into the trachea and secured tightly with a suture. The lungs were filled with 1-2 $\mathrm{mL}$ of dispase via the tracheal catheter and then allowed to collapse naturally, expelling part of the dispase. Low-melting-point agarose $\left(1 \%, 0.45 \mathrm{~mL}\right.$, stored in a $45^{\circ} \mathrm{C}$ water bath; Invitrogen, Paisley, UK) was slowly infused via the catheter. The lungs were immediately covered with crushed ice and incubated for $2 \mathrm{~min}$. Then, they were removed and placed into $12 \mathrm{~mL}$ polypropylene culture tubes with $2 \mathrm{~mL}$ of dispase (Sigma-Aldrich), incubated for $45 \mathrm{~min}$ at room temperature, and kept on ice until the next step. The lungs were transferred into $7 \mathrm{~mL}$ of Dulbecco's modified Eagle's medium (DMEM) with 0.01\% DNase I in $60 \mathrm{~mm}$ Petri dishes. The digested tissue was carefully separated from the airways with the curved edge of fine-tipped forceps and gently swirled for 5-10 $\mathrm{min}$. The resulting suspension was successively filtered through 100 and $40 \mu \mathrm{m}$ Falcon cell strainers and then through $25 \mu \mathrm{m}$ nylon mesh. The filtered suspension was centrifuged at $130 \times g$ for $8 \mathrm{~min}$ at $4{ }^{\circ} \mathrm{C}$ and resuspended in $10 \mathrm{~mL}$ of $10 \%$ fetal bovine serum (FBS) and $1 \%$ penicillin-streptomycin in $25 \mathrm{mM}$ 4-(2-hydroxyethyl)-1piperazineethanesulfonic acid (HEPES)-buffered DMEM.

\section{Magnetic purification of ATII cells from crude cell suspensions}

The cells were incubated with biotinylated anti-CD32 $(0.5 \mu \mathrm{g} /$ million cells; BD Pharmingen, San Diego CA, USA) and biotinylated anti-CD45 (1.5 $\mu \mathrm{g} /$ million cells; BD Pharmingen) for $30 \mathrm{~min}$ at $37^{\circ} \mathrm{C}$. Meanwhile, streptavidincoated magnetic particles (Thermo Fisher Scientific, Waltham, MA, USA) were washed twice in phosphate-buffered saline (PBS) (10 min each wash) in polypropylene culture tubes using a magnetic tube separator (Sigma-Aldrich). After incubation, the cells were centrifuged $(130 \times \mathrm{g}$ for $8 \mathrm{~min}$ at $4{ }^{\circ} \mathrm{C}$ ), resuspended in $7 \mathrm{~mL}$ of DMEM, added to the magnetic particles, and incubated with gentle rocking for $30 \mathrm{~min}$ at room temperature. At the end of the incubation, the tubes were attached to the magnetic tube separator with adhesive tape for $15 \mathrm{~min}$. The cell suspension was aspirated 
from the bottom of the tube using a narrow-stemmed transfer pipette, centrifuged, and resuspended in a culture medium. Cell viability was determined by trypan blue staining. The purity of alveolar type II cells was assessed via pro-SPC immunofluorescence staining. As in previous studies, $4-8 \times 10^{5}$ cells were isolated from a single mouse. In these samples, the purity of type II pneumocytes was 90-93\%. The isolated cells were maintained in 10\% FBS and $1 \%$ penicillin-streptomycin in $25 \mathrm{mM}$ HEPESbuffered DMEM.

\section{Fibroblast isolation and culture}

Primary human lung fibroblasts were obtained from SCH Biobank (Soonchunhyang Biobank, Bucheon, Korea) as previously described ${ }^{18}$. Briefly, the fibroblasts were derived from lung tissue obtained from IPF patients by video-assisted thoracoscopic biopsy. Lung samples were obtained by lung biopsy, usually 1 week after hospital admission. None of the patients had been treated with corticosteroids or immunosuppressive drugs at the time of the biopsy. Control fibroblasts were obtained from individuals who underwent a lobectomy to remove a primary lung tumor. No morphological evidence of disease was found in the tissue samples used for the isolation of control cells. Lung fibroblasts from IPF or control specimens were isolated by mechanical dispersal, and then trypsin digestion of tissues was used to mince them into $1 \mathrm{~mm}^{2}$ fragment. Fibroblast cultures were established in DMEM supplemented with $10 \%$ fetal calf serum, $100 \mathrm{U} / \mathrm{mL}$ penicillin, $100 \mathrm{mg} / \mathrm{mL}$ streptomycin, and $0.25 \mu \mathrm{g} / \mathrm{mL}$ amphotericin B. All cells were cultured at $37^{\circ} \mathrm{C}$ in $95 \%$ air $/ 5 \% \mathrm{CO}_{2}$ until just prior to reaching confluence, which generally occurred in 1-2 weeks. After three passages, immunoblotting was performed using anti-vimentin antibodies on adherent cells harvested from the same culture vessels. All cells showed the morphological characteristics of fibroblasts. All experiments with IPF and control fibroblasts were performed on cells before the sixth passage.

\section{Apoptosis assay}

Apoptotic cells in paraffin-embedded lung tissues were labeled using a terminal deoxynucleotidyl transferase (TdT)-mediated dUTP nick end labeling (TUNEL) assay kit (Roche Diagnostics, Basel, Switzerland). The numbers of TUNEL-positive (apoptotic) cells in three sections per sample were counted under a fluorescence microscope at $\times 400$ magnification (Carl Zeiss Microsystems, Thornwood, NY, USA) as described previously ${ }^{19}$. Mouse AECs were exposed to vehicle or $10 \mu \mathrm{g} / \mathrm{mL}$ BLM with spermidine for $24 \mathrm{~h}$ after overnight serum starvation. The dose of spermidine administered was $100 \mu \mathrm{M}$. An annexin VFITC/propidium iodide detection kit (BD Biosciences Pharmingen, San Diego, CA, USA) was used to determine the proportions of apoptotic and necrotic cells. Approximately, $1 \times 10^{6}$ cells $/ \mathrm{mL}$ were washed in PBS, surface stained, resuspended in binding buffer, incubated with FITC-conjugated annexin V and propidium iodide (PI) for $15 \mathrm{~min}$ in the dark at room temperature, washed, and resuspended in binding buffer.

\section{Immunoblotting}

Protein were extracted from cells with lysis buffer (\#78510; Thermo Fisher Scientific, Waltham, MA, USA) containing proteinase and phosphatase inhibitor cocktails (\#05892970001 and \#04906837001; Roche Diagnostics, Basel, Switzerland), and the samples were then centrifuged. Immunoblotting was performed as described previously. For each experiment, equal amounts of total protein were resolved by $10 \%$ sodium dodecyl sulfatepolyacrylamide gel electrophoresis. The proteins were transferred to a polyvinylidene difluoride membrane (\#ISEQ00010; Millipore, Billerica, MA, USA) and incubated with a specific primary antibody for $2 \mathrm{~h}$ at $37^{\circ} \mathrm{C}$ or for $24 \mathrm{~h}$ at $4{ }^{\circ} \mathrm{C}$. After being washed several times with PBS containing Tween, the membrane was incubated with anti-rabbit immunoglobulin G (IgG) horseradish peroxidase (HRP)-linked secondary antibody (\#7074; Cell Signaling Technology) or anti-mouse IgG HRP-linked secondary antibody (\#7076; Cell Signaling Technology), followed by chemiluminescence detection (\#34080; Thermo Fisher Scientific and \#1705061; BIO-RAD, Berkeley, CA, USA) with a ChemiDoc ${ }^{\mathrm{TM}}$ Touch Imaging System (BIO-RAD).

\section{SA- $\beta$-galactosidase staining}

Senescent cells were analyzed using senescenceassociated $\beta$-galactosidase (SA- $\beta$-gal) staining. Cells were grown in six-well plates, washed, fixed, and stained with the SA- $\beta$-gal cellular senescence assay kit (Cell Biolabs) according to the manufacturer's protocol and as previously described ${ }^{20}$. The sections were examined under a microscope.

\section{Immunofluorescence staining of fibroblasts}

Standard protocols for immunofluorescence microscopy were used as described previously ${ }^{21}$. Human primary fibroblasts were plated on cover-glass-bottom dishes and treated with or without with the indicated agents. The cells on the dishes were washed twice, fixed in $4 \%$ paraformaldehyde for $1 \mathrm{~h}$, and washed three times after fixation. The cells were prepared and stained with the indicated primary antibodies (1:50) overnight at $4{ }^{\circ} \mathrm{C}$. The slides were washed twice, incubated with chromogen-labeled secondary antibody (1:100) for $30 \mathrm{~min}$, and washed three times after being stained. Images were obtained with a confocal microscope (Zeiss LSM 510 META). Autophagosomes were identified as LC3B-positive puncta, and 
autophagolysosomes were identified by the coexpression of LC3B and LAMP-1.

\section{Animal model of BLM-induced lung fibrosis and treatment with spermidine}

Specific-pathogen-free C57BL/6 (Orient Bio Inc., Sungnam-Si, Gyeonggi-Do, Korea) mice were maintained under pathogen-free conditions. All animal procedures followed a protocol approved by the Institutional Animal Care and Use Committee of Soonchunhyang University Bucheon Hospital (SCHBC-animal-2016-011). On day 0, the mice were administered $3 \mathrm{U} / \mathrm{kg}$ BLM (Sigma-Aldrich) dissolved in a total volume of $200 \mu \mathrm{L}$ endotoxin-free water by intratracheal instillation. To determine the proper therapeutic dose of spermidine, we performed a pilot study with a small number of mice. We treated bleomycin-induced mice with $10,30,50$, and $100 \mathrm{mg} / \mathrm{kg}$ spermidine from day 10 to day 21, and then the analyzed inflammatory cells in BAL fluid. In addition, to evaluate the cellular toxicity of spermidine, $\mathrm{LDH}$ levels in BAL fluid were measured. We found that $50 \mathrm{mg} / \mathrm{kg}$ spermidine significantly diminished neutrophils and reduced LDH levels in the BAL fluid, indicating that $50 \mathrm{mg} / \mathrm{kg}$ spermidine was both effective and safe in mice (Supplementary Fig. 1). On days 10-21, the mice were administered $50 \mathrm{mg} / \mathrm{kg}$ spermidine (Sigma-Aldrich) dissolved in endotoxin-free water containing $0.3 \%$ dimethyl sulfoxide by intraperitoneal instillation. The sham control mice were treated with PBS only. On day 21, the mice were sacrificed with an overdose of a ketamine/xylazine mixture, and bronchoalveolar lavage (BAL) was performed by instilling $1 \mathrm{~mL}$ of PBS, which was gently retrieved, four times as described previously ${ }^{22}$. Cell numbers were measured using a hemocytometer, and differential cell counts were performed on slides prepared by cytocentrifugation and Diff-Quik staining (E. Merck KG, Darmstadt, Germany). The BAL fluid was centrifuged at $500 \times g$ for $10 \mathrm{~min}$, and the supernatant was stored at $-70{ }^{\circ} \mathrm{C}$. Some of the cell-free supernatants were used for biochemical analyses, such as lactate dehydrogenase (LDH) assays. The Institutional Animal Care and Use Committee of Soonchunhyang University Bucheon Hospital approved this study (SCHBC-2016-012).

\section{Histological assays}

A portion of each left lung was fixed in $4 \%(\mathrm{v} / \mathrm{v})$ buffered paraformaldehyde and embedded in paraffin. The tissue was cut into $5 \mu \mathrm{m}$-thick slices and stained with hematoxylin and eosin (H\&E) or Masson's trichrome. The right lung was snap-frozen by immersion in liquid nitrogen and stored at $-80{ }^{\circ} \mathrm{C}$ prior to RNA and protein extraction. Lung sections were stained with H\&E for histopathological analyses or with Masson's trichrome to evaluate collagen content and distribution. The Ashcroft score for the evaluation of lung fibrosis has previously been described $^{23}$.

\section{Immunohistochemical staining}

The lung tissues were dehydrated and embedded in paraffin. For histological examination, $4 \mu \mathrm{m}$-thick sections on slides were treated with $1.4 \% \mathrm{H}_{2} \mathrm{O}_{2}$-methanol for $30 \mathrm{~min}$ to block endogenous peroxidase. Then, nonspecific binding was blocked with $1.5 \%$ normal saline, and the slides were incubated with rabbit anti-p21 (1:200; \#ab188224; Abcam, Cambridge, UK) and rabbit anti-p16 (1:100; \#ab51243; Abcam) antibodies. The next day, the sections were incubated with $\mathrm{ABC}$ kit reagents (Vector Laboratories, Burlingame, CA, USA). The color reaction was developed by staining with a liquid DAB + substrate kit (Golden Bridge International Inc., Mukilteo, WA, USA). After immunohistochemical staining, the slides were counterstained with Harris's hematoxylin for $1 \mathrm{~min}$.

\section{Masson's trichrome staining}

The mouse lung sections were placed in Bouin's solution at $56^{\circ} \mathrm{C}$ for $1 \mathrm{~h}$ and then stained successively with Mayer's hematoxylin solution for $5 \mathrm{~min}$, Biebrich scarletacid fuchsin solution for $10 \mathrm{~min}$, phosphomolybdic acidphosphotungstic acid for $15 \mathrm{~min}$, and aniline blue for $2 \mathrm{~h}$ (staining reagents from Sigma-Aldrich). The sections were examined under a microscope.

\section{Measurement of hydroxyproline in the mouse lung}

To estimate the amount of collagen in the lung, the right lungs were used in a hydroxyproline assay (\#MAK008; Sigma-Aldrich) according to the manufacturer's protocol. Briefly, the lungs were weighed, homogenized in sterile water, and hydrolyzed in $12 \mathrm{~N} \mathrm{HCl}$ at $120{ }^{\circ} \mathrm{C}$ for $3 \mathrm{~h}$. The hydrolyzed samples were incubated with 4-(dimethylamino) benzaldehyde for $90 \mathrm{~min}$ at $60{ }^{\circ} \mathrm{C}$, and the absorbance of oxidized hydroxyproline was measured at $560 \mathrm{~nm}$. The amount of collagen is expressed in micrograms per milligram of lung tissue.

\section{Measurement of proinflammatory and profibrotic cytokine levels}

ELISA kits were used to measure the concentrations of IL- $1 \beta$, TNF- $\alpha$, and the active form of TGF- $\beta 1$ in lung tissue (IL-1 $\beta$ : \#88701322; eBioscience, Inc., TNF- $\alpha$ : \#88732422; eBioscience, Inc., TGF- $\beta 1$ : \#DY1679; R\&D Systems.) according to the manufacturers' instructions.

\section{Statistical analyses}

All data are expressed as the means \pm standard error. The data were analyzed using the Kruskal-Wallis test, followed by the Mann-Whitney $U$ test (with Bonferroni correction for intergroup comparisons), and $p$ values $<$ 0.05 were considered significant. 


\section{Results}

\section{Spermidine attenuates BLM-induced cell death in AECs}

BLM generates reactive oxygen species, which are responsible for the induction of cell death, and BLM is generally administered to establish lung fibrosis models for in vivo studies ${ }^{24}$. We examined the effects of spermidine on cell death in primary mouse AECs induced by BLM in an in vitro system. The selected concentrations of spermidine used in this experiment did not show any significant cytotoxicity at a concentration of $100 \mu \mathrm{M}$ for up to $48 \mathrm{~h}$ of incubation, and cell viability remained $>95 \%$ as measured using the MTT assay (data not shown). Twenty-four hours after BLM $(10 \mu \mathrm{g} / \mathrm{mL})$ administration in the presence of spermidine $(100 \mu \mathrm{M})$ or vehicle, the proportions of apoptotic and necrotic cells were assessed by flow cytometry (Fig. 1). Spermidine significantly diminished BLM-induced increases in the fractions of apoptotic and necrotic cells (Fig. 1). These data indicated that spermidine significantly attenuated BLM-induced AEC apoptosis and necrosis.

\section{Spermidine inhibits BLM-induced senescence in AECs and mouse lungs}

Cellular senescence is a conserved state of stable replicative arrest induced by aging-related stressors, including oxidative stress, DNA damage, and telomerase attrition ${ }^{25}$. Previous studies have shown that BLM, a fibrosisinducing agent, induces cellular senescence ${ }^{26}$. Further evaluation by measuring SA- $\beta$-gal activity in cultured mouse AECs showed that BLM-induced SA- $\beta$-gal-positive staining was almost abolished by spermidine treatment (Fig. 2a). Next, we evaluated the effects of spermidine on BLM-induced senescence in mouse lung tissue. Immunoblot analyses showed that p16 and p21, which are molecular markers of senescence ${ }^{27,28}$, were increased in response to BLM administration compared to PBS treatment (Fig. 2b). Treatment with spermidine significantly downregulated the BLM-induced increases in p16 and p21 levels (Fig. 2b). Immunohistochemical analysis of mouse lung sections also showed that the BLMinduced increases in p16 and p21 were attenuated by spermidine (Fig. 2c). These results suggest that spermidine effectively inhibits the activation of senescence in BLM-induced AECs.

\section{Exogenous spermidine attenuates BLM-induced lung inflammation and fibrosis development}

To clarify the therapeutic effects of spermidine on established BLM-induced lung fibrosis, we used an established BLM-induced lung fibrosis model as previously described with modifications ${ }^{29}$. Because excessive doses of spermidine are toxic to mammalian cells ${ }^{10}$, we treated BLM-induced mice with 10, 30, 50, and $100 \mathrm{mg} / \mathrm{kg}$ spermidine and measured BAL cell counts and LDH levels (Supplementary Fig. 1). BAL cell counts were measured, $50 \mathrm{mg} / \mathrm{kg}$ spermidine most dramatically decreased neutrophil numbers compared to those of the other groups (Supplementary Fig. 1a). LDH levels in BAL fluid were dose-dependently decreased by spermidine; however, $100 \mathrm{mg} / \mathrm{kg}$ spermidine increased LDH levels (Supplementary Fig. 1b). This result suggests that spermidine may cause cellular toxicity in certain doses in vivo. BLM was administered via the trachea on day 0 , spermidine $(50 \mathrm{mg} /$ $\mathrm{kg}$ ) was administered intraperitoneally on days $10-21$, and lung samples were evaluated on day 21 .

H\&E staining showed that spermidine effectively reversed BLM-induced distortions in lung structure (Fig. 3a). Consistent with the H\&E staining results, BAL fluid analyses showed that spermidine significantly attenuated lung inflammation and reduced the total numbers of macrophages, neutrophils, and lymphocytes (Fig. 3b). Furthermore, spermidine treatment clearly reduced lung

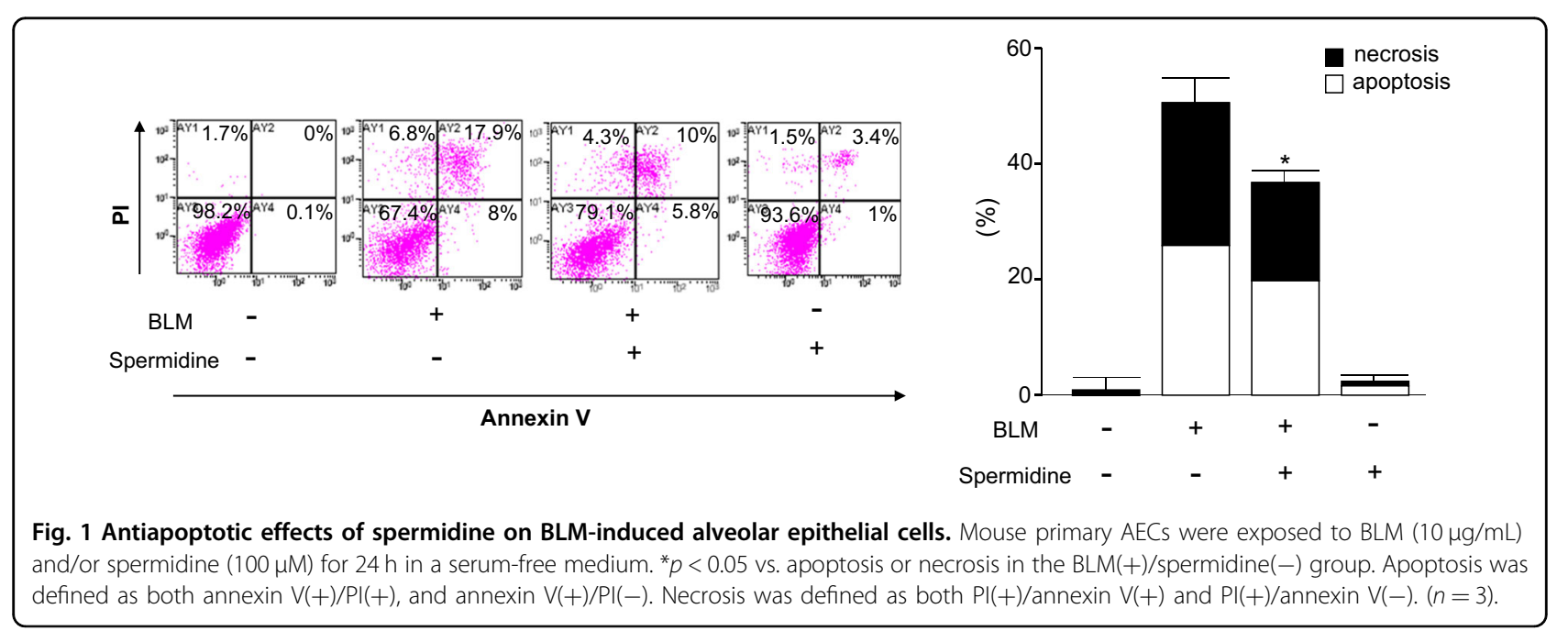




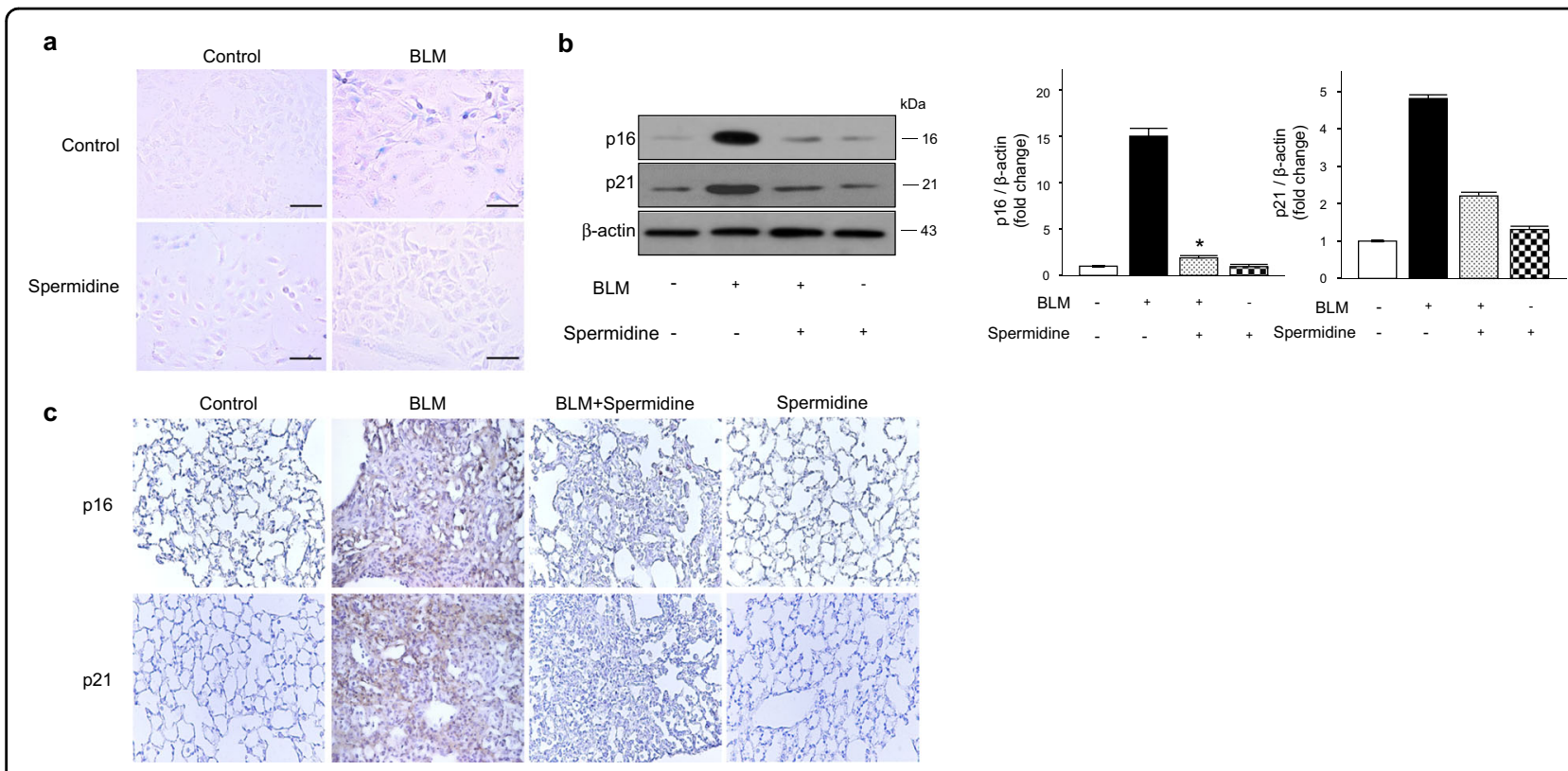

Fig. 2 Spermidine inhibits senescence in BLM-induced mouse lung and alveolar epithelial cells. a. Mouse primary AECS were exposed to BLM $(10 \mu \mathrm{g} / \mathrm{mL})$ and/or spermidine $(100 \mu \mathrm{M})$ for $24 \mathrm{~h}$ in serum-free medium. $\beta$-gal staining showed increased SA- $\beta$-gal activity in AECS after BLM exposure, and treatment with $100 \mu \mathrm{M}$ spermidine reversed senescence in these cells. Scale bar, $50 \mu \mathrm{m}$. Mice were treated with BLM (3 U/kg) intratracheally on day 0 , and spermidine $(50 \mathrm{mg} / \mathrm{kg} / \mathrm{mouse} /$ day) was administered intraperitoneally on days 10-21. Lung samples were collected on day 21 .

b Immunoblot analyses of the expression of p16 and p21 in whole lungs from mice. The intensity of each band was quantified by densitometry, and the data were normalized to $\beta$-actin. ${ }^{*} p<0.05$ vs. the BLM(+)/spermidine(-)group. $(n=3)$ c Photographs of immunohistochemical staining showing the expression of p16 and p21 in mouse lungs. Original magnification $\times 100$.

fibrosis caused by collagen deposition, as indicated by Masson's trichrome staining and Ashcroft scoring (Fig. 3c, d). The hydroxyproline assay further confirmed the antifibrotic effect of spermidine on BLM-induced fibrosis (Fig. 3e).

\section{Exogenous spermidine inhibits BLM-induced increases in} profibrotic and proinflammatory mediators in mouse lungs

To determine whether spermidine inhibits the BLMinduced increases in proinflammatory cytokines and fibrotic mediators produced by macrophages and neutrophils, the protein levels of interleukin-1 $\beta$ (IL-1 $\beta$ ), TNF- $\alpha$, and active TGF- $\beta 1$ in lung lysates were measured. All of the measured proinflammatory mediators and TGF- $\beta 1$ in spermidinetreated mice were significantly decreased compared to those in BLM-induced mice (Fig. 4).

\section{Exogenous spermidine decreases the expression of ERS- related proteins in mouse lungs}

Next, we investigated whether ERS was attenuated by spermidine-mediated protection against BLM-induced lung injury/fibrosis. ERS-related molecules in lung lysates were measured by immunoblotting. BLM-induced increases in ER-related proteins, including CHOP, GRP78, ATF6, and IRE-1, were also inhibited by spermidine (Fig. 5). This finding demonstrates that exogenous spermidine reduced BLM-induced lung injury/fibrosis and was associated with downregulation of the ERS pathway.

\section{Exogenous spermidine protects against BLM-induced apoptosis in mouse lungs}

The ability to respond to perturbations in ER function is critical for cell survival, but chronic or unresolved ERS can lead to apoptosis ${ }^{30}$. First, we evaluated whether BLMinduced apoptotic cell death was mediated by excessive ERS. The ERS inhibitor 4-PBA was administered with BLM to mouse AECs, and apoptosis was measured by annexinV/PI staining. As expected, the BLM-induced increases in apoptotic cell proportions were significantly decreased by 4-PBA (Supplementary Fig. 2). To determine whether spermidine inhibited BLM-induced apoptosis in vivo, we measured caspase- 3 levels in mouse lungs. The protein levels of active caspase- 3 were increased by BLM compared to those of the controls. The protein levels of the active form of caspase-3 in spermidine-treated mice were significantly decreased compared to those of BLM-induced mice (Fig. 6a). Similarly, a TUNEL assay revealed that approximately $75 \%$ of fewer apoptotic cells were present in the lungs of spermidine-treated mice than in the lungs of BLM-induced mice (Fig. 6b). 


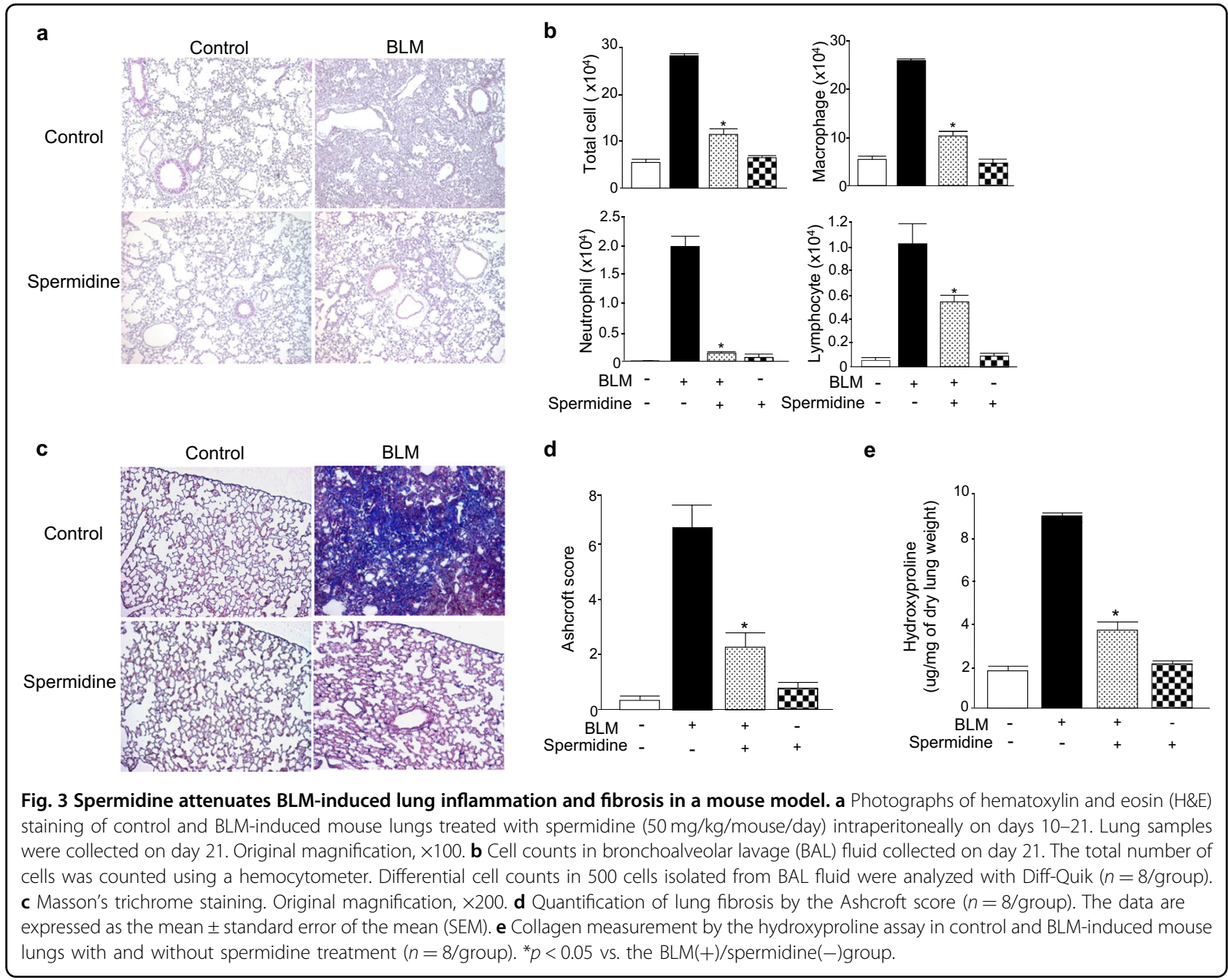

a

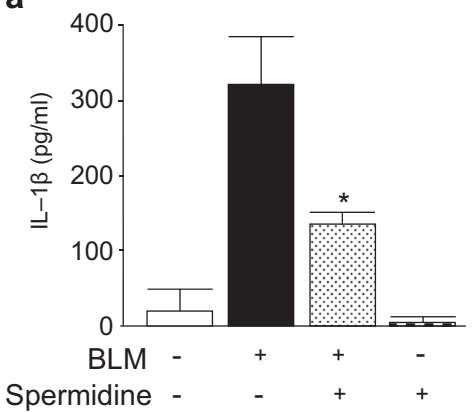

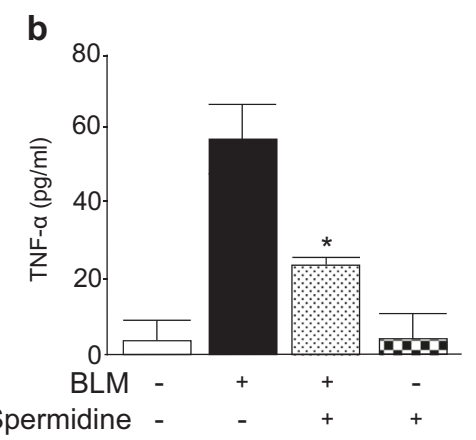

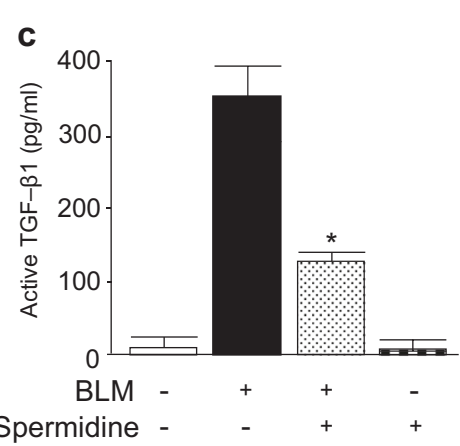

Fig. 4 Spermidine inhibits BLM-induced increases in proinflammatory mediators and profibrotic cytokines in mouse lungs. a $\mid L-1, \mathbf{b} T N F-a$, and $\mathbf{c}$ active TGF- $\beta 1$ protein expression were measured using ELISA. The data are expressed as the mean \pm SEM. ${ }^{*} p<0.05$ vs. the BLM $(+) /$ spermidine (-)group.

\section{Exogenous spermidine enhances autophagy in IPF fibroblasts and BLM-induced lungs}

Lung fibroblasts are the principal components of the interstitium and the main producers of the ECM, and the proliferation of (myo)fibroblasts is the main pathological finding in $\mathrm{IPF}^{31}$. Recent reports have implicated defective fibroblast autophagy in the pathogenesis of $\mathrm{IPF}^{32,33}$. We investigated whether spermidine regulated autophagic 


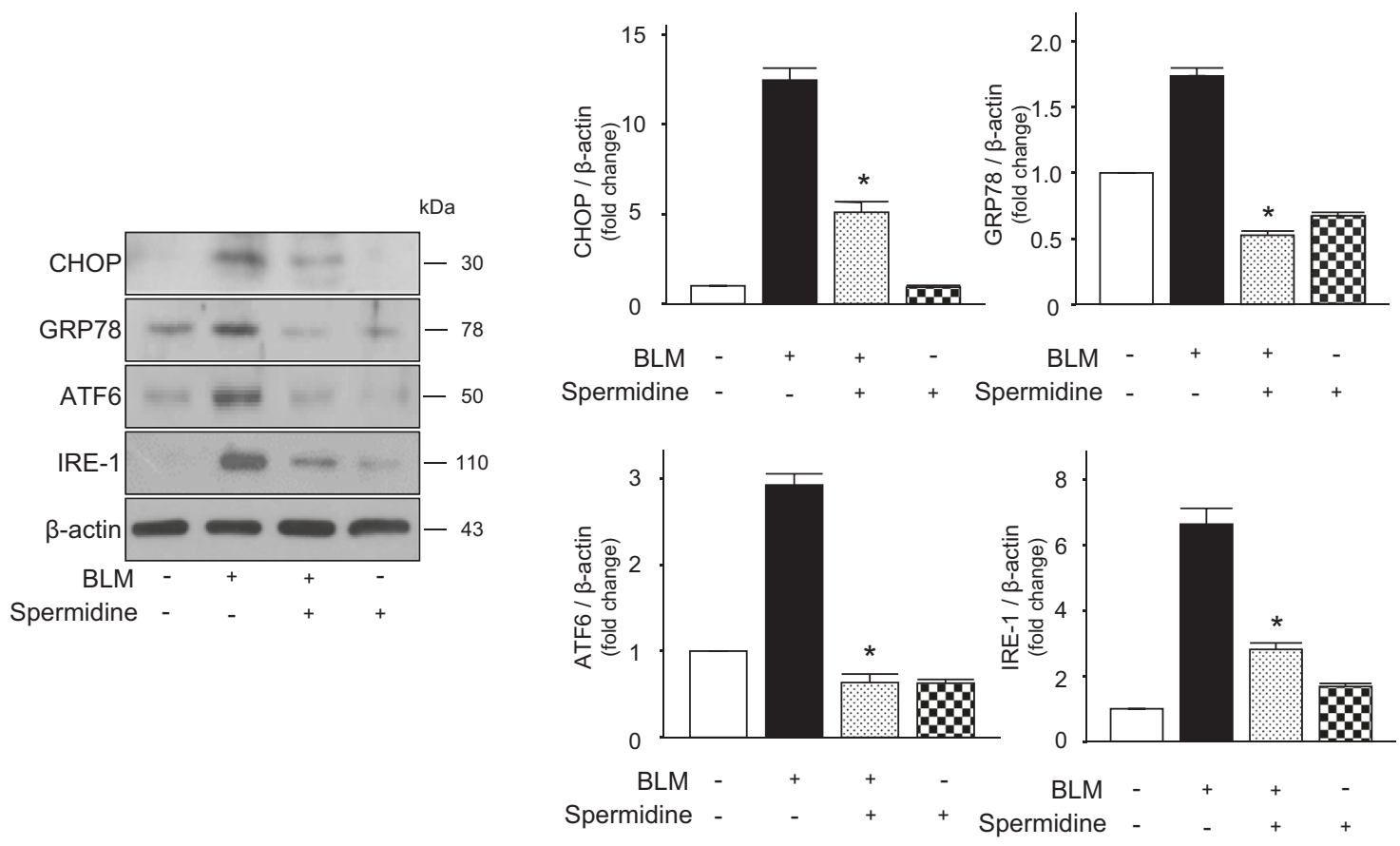

Fig. 5 Spermidine decreases the expression of ERS-related proteins. The protein expression of CHOP, GRP78, ATF6, and IRE-1 was measured by immunoblotting. The intensity of each band was quantified by densitometry, and the data were normalized to $\beta$-actin. The data are expressed as the mean \pm SEM. ${ }^{*} p<0.05$ vs. the BLM(+)/spermidine(-)group. $(n=3)$.

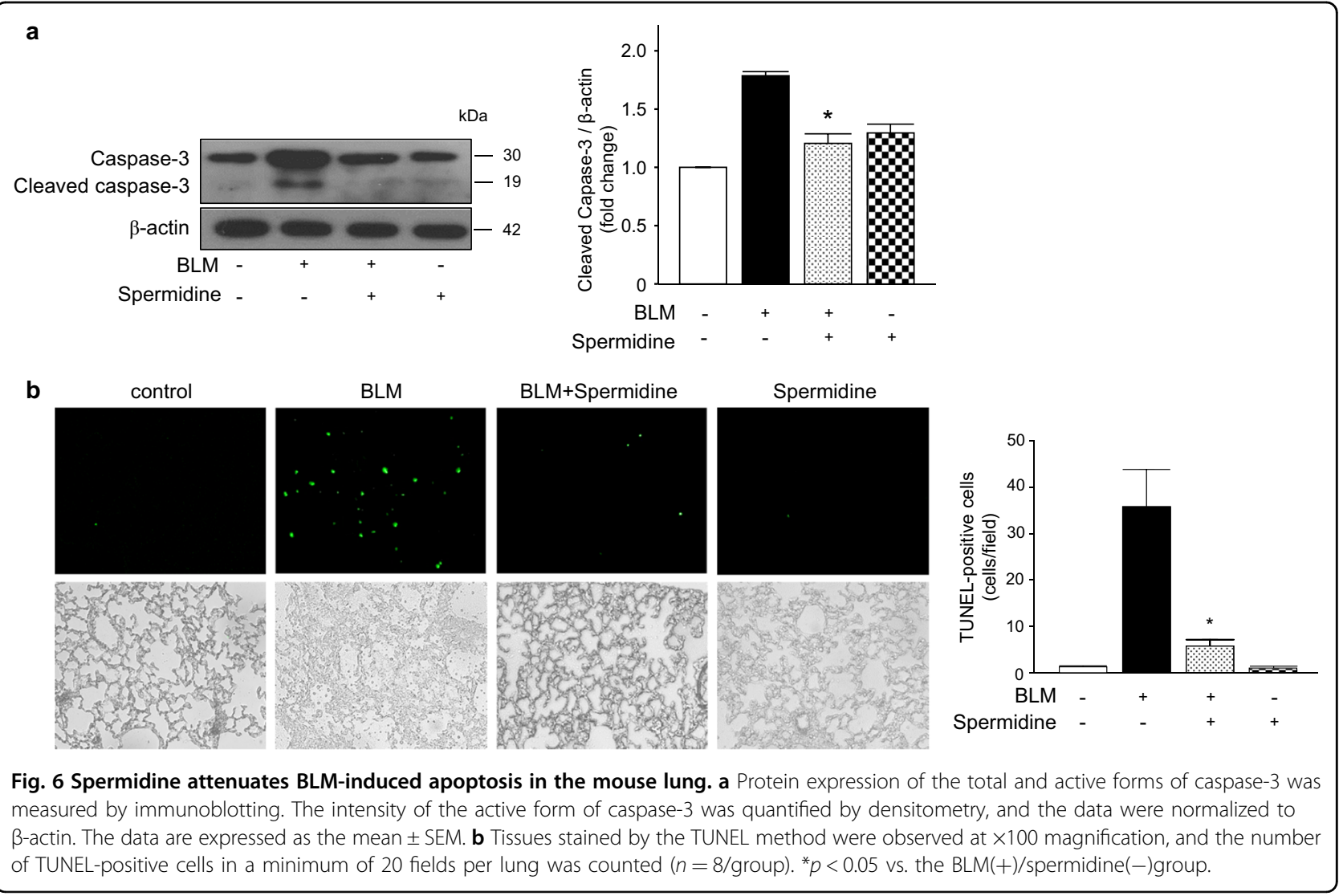




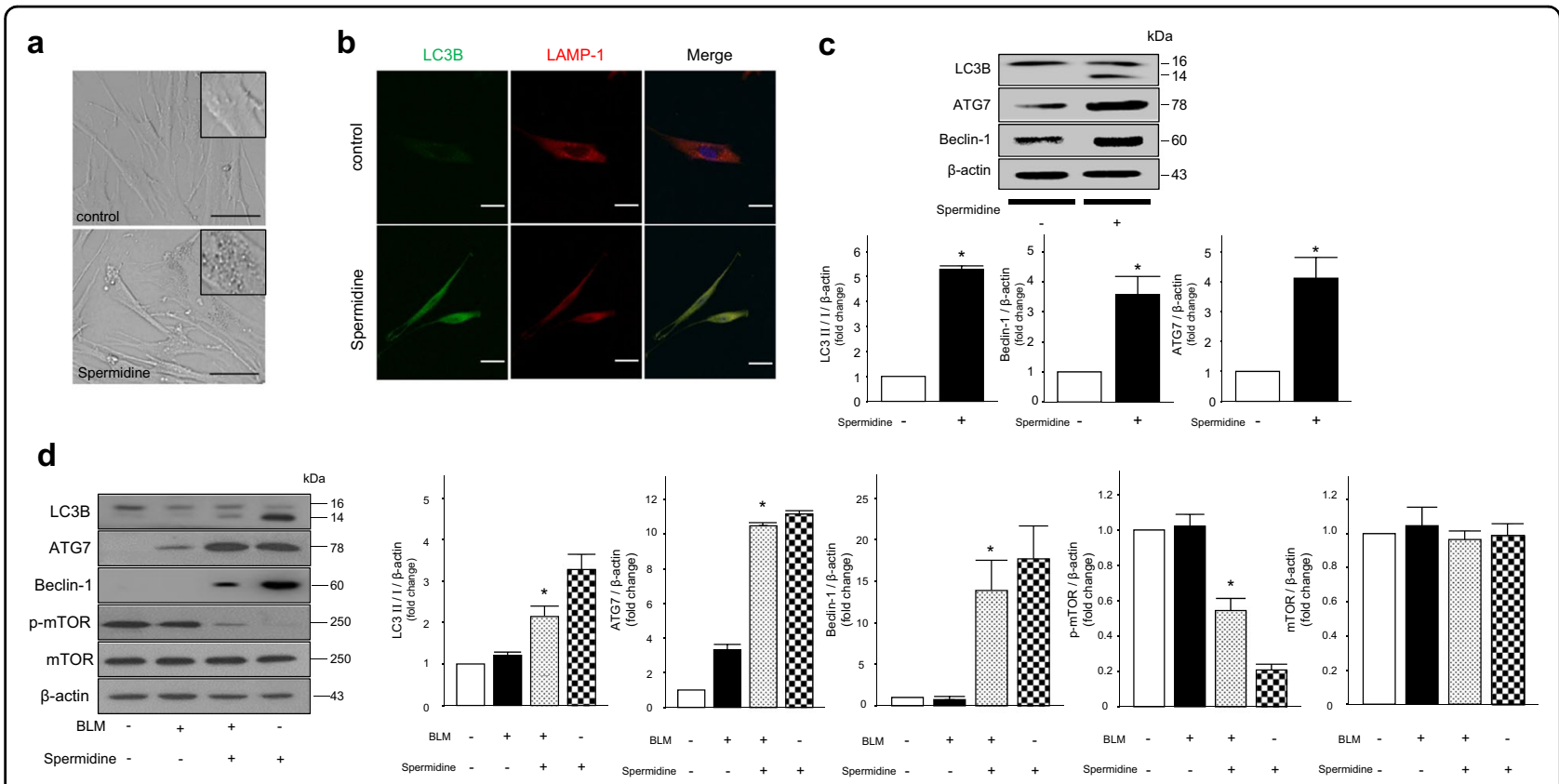

Fig. 7 Spermidine enhances autophagy in idiopathic pulmonary fibrosis (IPF) fibroblasts and BLM-induced mouse lungs. IPF fibroblasts were treated with spermidine $(100 \mu \mathrm{M})$ for $6 \mathrm{~h}$. a Light microscopy images showing spermidine-induced vacuolar changes in fibroblasts; $\times 400$, inset: $\times 1000$. Scale bars, $50 \mu \mathrm{m}$. b Laser confocal microscopy was performed to measure LC3B and LAMP-1 by indirect immunofluorescence. Scale bars, $5 \mu \mathrm{m}$. c LC3B, ATG7, and beclin-1 expression was evaluated by immunoblotting. Densitometric analyses of independent experiments were performed. ${ }^{*} p<0.05$ vs. the spermidine ( - ) group. The data are expressed as the mean \pm SEM $(n=4)$. BLM-induced mice were treated with spermidine $(50 \mathrm{mg} / \mathrm{kg} / \mathrm{mouse} /$ day) intraperitoneally on days 10-21. Lung samples were collected on day 21. $\mathbf{d}$ The expression of autophagy-related proteins, including LC3 I/II, ATG7, beclin-1, mTOR, and p-mTOR, in lung lysates was measured by immunoblotting. Densitometric analyses of the experiments ( $n=6 /$ group). ${ }^{*} p<0.05$ vs. the $\mathrm{BLM}(+) /$ spermidine(-)group.

flux in IPF fibroblasts. First, spermidine $(100 \mu \mathrm{M})$ led to the formation of cytoplasmic vacuoles after $6 \mathrm{~h}$ of treatment, suggesting the possible involvement of autophagy (Fig. 7a). The conversion of LC3-I to LC3-II is a hallmark of autophagy ${ }^{34}$. In addition, ATG7 and beclin-1 are key mediators of the canonical autophagy pathway ${ }^{35}$. We evaluated LC3B I/II expression in IPF fibroblasts by immunofluorescence and immunoblotting. Spermidine increased LC3B-expressing fibroblasts, as shown by immunofluorescence microscopy (Fig. 7b). Spermidine also increased the LC3B-I/II ratio and enhanced ATG7 and beclin-1 expression in fibroblasts (Fig. 7c). These data suggest that spermidine activates autophagy via a canonical pathway. In BLM-induced lungs, spermidine also enhanced the LC3 I/II ratio and the expression of ATG7 and beclin-1; however, p-mTOR expression decreased (Fig. 7d). These findings demonstrate that spermidine activates autophagy and that this effect may be beneficial in fibrotic lungs.

\section{Discussion}

We showed that treatment with spermidine inhibited BLM-induced increases in AEC death, which is composed of the main pathophysiology of IPF. Our findings indicate that spermidine protects against BLM-induced lung inflammation and fibrosis. These beneficial effects are associated with suppressed upregulation of proinflammatory cytokines and neutrophilic inflammation and the prevention of apoptotic death in the lungs. In addition, we found other favorable effects of spermidine, particularly in the context of IPF pathogenesis, including the inhibition of cellular senescence, attenuation of ERS-mediated apoptosis, and activation of autophagy in primary lung fibroblasts and in vivo. This study is the first to report the therapeutic effects of spermidine on BLM-mediated experimental lung fibrosis and to elucidate the possible mechanism.

Senescence is a state of irreversible cell cycle arrest accompanied by an abnormal secretory profile and is thought to play a critical role in both development and wound repair ${ }^{36}$. As the incidence of IPF continuously increases with age, age-related mechanisms such as cellular senescence and telomere shortening have been proposed to be pathogenic drivers of lung fibrosis ${ }^{36,37}$. Recently, published reports have suggested that accelerated AEC senescence may contribute to the pathogenesis of $\mathrm{IPF}^{38,39}$. We showed that exogenous spermidine suppresses BLM-induced increases in cellular senescence in primary mouse AECs and in vivo (Fig. 2a). This result confirms that spermidine has antisenescence activity against oxidative stressors such as BLM. 
The ER is an intracellular organelle that is responsible for the folding and sorting of proteins ${ }^{40}$. Various conditions, such as hypoxia, ischemia, and oxidative stress, can impair ER function and cause the accumulation of unfolded or misfolded proteins, resulting in ER stress, which leads to the activation of a signal response known as the unfolded protein response (UPR) ${ }^{41}$. According to previous reports, ER stress and UPR activation have been observed in the alveolar epithelium of patients with $\mathrm{IPF}^{42,43}$. Recently, ER stress and UPR have been linked to the development of lung fibrosis through the regulation of AEC apoptosis, epithelial-mesenchymal transition, and myofibroblast differentiation ${ }^{44,45}$. Previous studies have shown that spermidine acts as an inhibitor of ER stress in vitro and in vivo ${ }^{46,47}$. Our study indicates that spermidine also alleviates the BLM-induced increases in ATF6, IRE-1, GRP78, and CHOP in the lungs (Fig. 5). These results suggest that spermidine inhibits pulmonary ER stress and the activation of UPR signaling in BLMinduced lung fibrosis. Spermidine-mediated protection against BLM-induced lung fibrosis might be associated with the downregulation of ER stress in the lungs.

Polyamines have been implicated in apoptotic cell death in numerous studies in which cellular polyamines were altered either by overexpression or by the inhibition of biosynthetic enzymes ${ }^{48}$ and in studies with cells or animals with genetically modified polyamine pathways ${ }^{49}$. Either excessive accumulation or depletion of cellular polyamines is harmful to mammalian cells and can lead to cell death ${ }^{48}$. Polyamines may activate or inhibit apoptosis depending on the concentration and the specific apoptotic inducers. Regarding the possible mechanisms of the antiapoptotic effects, polyamine binding to DNA protects against DNA cleavage from ionizing radiation ${ }^{50}$; polyamines may also act as scavengers of reactive oxygen radicals ${ }^{51}$. However, excessive polyamines or the activation of amine oxidation can also cause oxidative stress and apoptosis by the generation of $\mathrm{H}_{2} \mathrm{O}_{2}$ and reactive aldehydes in cells ${ }^{10,52}$. In this study, $100 \mu \mathrm{M}$ spermidine showed a beneficial effect by attenuating BLM-induced apoptosis in vitro (Fig. 1). We also found that although this concentration was not fatal, $100 \mathrm{mg} / \mathrm{kg}$ spermidine was toxic to BLM-induced mice after repeated administrations (Supplementary Fig. 1). These data suggest that the accumulation of excessive spermidine may be harmful both in vitro and in vivo.

When ER stress is elevated, cell apoptosis can be stimulated by the activation of caspase-12, which is then released into the cytoplasm, where it activates the final apoptotic pathway to sequentially activate caspase- 9 and caspase-3. Our findings showed decreases in caspase-3 protein expression (Fig. 6a) and the number of TUNELpositive cells suggests that spermidine inhibits BLMinduced apoptosis in the lung (Fig. 6b). To further determine whether ER stress was involved in BLMinduced apoptosis, we treated primary AECs with 4-PBA, a selective inhibitor of ERS, as previously described ${ }^{53}$. Treatment significantly reduced the proportion of apoptotic cells, which confirmed that the effects of spermidine occurred through ER-induced cell apoptosis (Supplementary Fig. 2).

Autophagy is a fundamental homeostatic process used by cells to degrade and recycle cellular proteins and remove damaged organelles. Autophagy is activated in response to intracellular or extracellular factors, such as hypoxia, ERS or oxidative stress, organelle damage, and pathogen infection ${ }^{54}$. Because spermidine is a physiological autophagy inducer ${ }^{55}$, we evaluated whether it reversed autophagy impairment in IPF fibroblasts and in vivo. Indeed, we found that spermidine enhanced the formation of autophagosomes by activating the expression of key autophagic molecules, such as LC3-II, beclin-1, and ATG7, in IPF fibroblasts and BLM-induced fibrotic lung tissues (Fig. 7). Spermidine also suppressed mTOR, a downstream mediator of PI3K/AKT signaling that inhibits macroautophagy ${ }^{56}$ and is part of an ATG7-dependent canonical pathway (Fig. 7d). These data confirm that spermidine activates autophagy in IPF fibroblasts and experimental lung fibrosis. One study demonstrated impaired autophagy in the lung tissues of patients with IPF, as indicated by p62 accumulation and decreased LC-II expression ${ }^{33}$. Decreased autophagy-mediated clearance of ECM proteins leads to their accumulation, contributing to the progression of fibrosis. Recently, Kim et al. reported that the induction of autophagy alleviates BLM-induced lung fibrosis ${ }^{21}$. Thus, spermidine-mediated enhancement of autophagy may help resolve fibrosis.

Spermidine is a ubiquitous polycation that is associated with several important cellular functions and helps to maintain general cell homeostasis. Polyamines are essential for cell growth and proliferation and tissue regeneration. Polyamines bind and stabilize DNA and RNA, have antioxidant activities, modulate enzyme functions and are required for the regulation of translation ${ }^{57}$. Spermidine acts as a natural autophagy inducer and antiaging compound and exhibits many beneficial effects, such as cardioprotective, neuroprotective, and antitumorigenic effects ${ }^{14}$. In polyamine metabolism, spermidine is formed from its precursor putrescin or by the degradation of spermine, and its biosynthesis is connected to arginine and nitric oxide metabolism via ornithine ${ }^{58}$. North et al. reported increased airway spermidine levels in asthma and an association with enhanced airway hyperresponsiveness through the inhibition of constitutive $\mathrm{NO}$ synthesis ${ }^{59}$. To the best of our knowledge, only two studies have examined the levels of spermidine in lung tissues with IPF using mass spectrometry. We previously found that spermidine levels were $30 \%$ lower in IPF lungs 
than in controls (Supplementary Fig. 3) ${ }^{16}$. However, Zhao et al. reported increased spermidine levels in IPF lungs ${ }^{60}$. We hypothesize that this discrepancy may be due to different sample numbers and to the different stages of IPF in lung tissue. This study is the first to report the effects of exogenous spermidine on experimental lung fibrosis and to investigate its possible mechanism.

In conclusion, exogenous spermidine administration attenuated lung inflammation and fibrosis in established BLM-induced experimental fibrosis. These findings were associated with decreased cellular senescence, ER stressrelated apoptosis in AECs, and the induction of autophagy. Taken together, these data suggest that spermidine may be a promising therapeutic agent for fibrotic lung diseases, including IPF.

The English in this document has been checked by at least two professional editors, both of whom are native speakers of English. For a certificate, please see: http:// www.textcheck.com/certificate/rFdygU.

\section{Acknowledgements}

This work was supported by the Basic Science Research Program through the National Research Foundation of Korea (NRF) funded by the Ministry of Science, ICT (Information and Communication Technologies), and Future Planning [NRF-2019R1A2C1006351] and supported by the Soonchunhyang University Research Fund.

\section{Author contributions}

S.W.P. designed the research; J.H. and K.S.S. performed the experimental work; S.W.P., A.R.B., and S.S.C. conducted data analysis and wrote the paper; and A.S.J. and D.J.K. collected the samples and provided the reagents.

\section{Conflict of interest}

The authors declare that they have no conflict of interest.

\section{Publisher's note}

Springer Nature remains neutral with regard to jurisdictional claims in published maps and institutional affiliations.

Supplementary information accompanies this paper at https://doi.org/ 10.1038/s12276-020-00545-z.

Received: 25 August 2020 Revised: 1 November 2020 Accepted: 3 November 2020.

Published online: 14 December 2020

\section{References}

1. American Thoracic Society/European Respiratory Society International Multidisciplinary Consensus Classification of the Idiopathic Interstitial Pneumonias. This joint statement of the American Thoracic Society (ATS), and the European Respiratory Society (ERS) was adopted by the ATS board of directors, June 2001 and by the ERS Executive Committee, June 2001. Am. J. Respir. Crit. Care Med. 165, 277-304 (2002).

2. Visscher, D. W. \& Myers, J. L. Histologic spectrum of idiopathic interstitial pneumonias. Proc. Am. Thorac. Soc. 3, 322-329 (2006).

3. Selman, M. \& Pardo, A. Role of epithelial cells in idiopathic pulmonary fibrosis: from innocent targets to serial killers. Proc. Am. Thorac. Soc. 3, 364-372 (2006).

4. Sisson, T. H. et al. Targeted injury of type II alveolar epithelial cells induces pulmonary fibrosis. Am. J. Respir. Crit. Care Med. 181, 254-263 (2010).

5. Lawson, W. E. et al. Endoplasmic reticulum stress enhances fibrotic remodeling in the lungs. Proc. Natl Acad. Sci. USA 108, 10562-10567 (2011).
6. Tanjore, H., Blackwell, T. S. \& Lawson, W. E. Emerging evidence for endoplasmic reticulum stress in the pathogenesis of idiopathic pulmonary fibrosis. Am. J. Physiol. Lung Cell. Mol. Physiol. 302, L721-L729 (2012).

7. Stuart, B. D. et al. Effect of telomere length on survival in patients with idiopathic pulmonary fibrosis: an observational cohort study with independent validation. Lancet Respir. Med. 2, 557-565 (2014).

8. Faner, R., Rojas, M., Macnee, W. \& Agusti, A. Abnormal lung aging in chronic obstructive pulmonary disease and idiopathic pulmonary fibrosis. Am. J. Respir. Crit. Care Med. 186, 306-313 (2012).

9. Tabor, C. W. \& Tabor, H. Polyamines. Annu. Rev. Biochem. 53, 749-790 (1984).

10. Pegg, A. E. \& Casero, R. A. Jr Current status of the polyamine research field. Methods Mol. Biol. 720, 3-35 (2011).

11. Igarashi, K. \& Kashiwagi, K. Modulation of cellular function by polyamines. Int. J. Biochem. Cell Biol. 42, 39-51 (2010).

12. Pegg, A. E. Mammalian polyamine metabolism and function. IUBMB Life $\mathbf{6 1}$, 880-894 (2009).

13. Gerner, E. W. \& Meyskens, F. L. Jr. Polyamines and cancer: old molecules, new understanding. Nat. Rev. Cancer 4, 781-792 (2004).

14. Madeo, F., Eisenberg, T., Pietrocola, F. \& Kroemer, G. Spermidine in health and disease. Science 359, eaan2788 (2018)

15. Eisenberg, T. et al. Induction of autophagy by spermidine promotes longevity. Nat. Cell Biol. 11, 1305-1314 (2009).

16. Kang, Y. P. et al. Metabolic profiling regarding pathogenesis of idiopathic pulmonary fibrosis. J. Proteome Res. 15, 1717-1724 (2016).

17. Corti, M., Brody, A. R. \& Harrison, J. H. Isolation and primary culture of murine alveolar type II cells. Am. J. Respir. Cell Mol. Biol. 14, 309-315 (1996).

18. Uhal, B. D. et al. Fibroblasts isolated after fibrotic lung injury induce apoptosis of alveolar epithelial cells in vitro. Am. J. Physiol. 269, L819-L828 (1995).

19. Lee, E. et al. Overexpression of apolipoprotein $A 1$ in the lung abrogates fibrosis in experimental silicosis. PloS ONE 8, e55827 (2013).

20. Kim, K. S., Kang, K. W., Seu, Y. B., Baek, S. H. \& Kim, J. R. Interferon-gamma induces cellular senescence through p53-dependent DNA damage signaling in human endothelial cells. Mech. Ageing Dev. 130, 179-188 (2009).

21. Kim, M. S. et al. IL-37 attenuates lung fibrosis by inducing autophagy and regulating TGF- $\beta 1$ production in mice. J. Immunol. 203, 2265-2275 (2019).

22. Kim, T. H. et al. Role of lung apolipoprotein A-I in idiopathic pulmonary fibrosis: antiinflammatory and antifibrotic effect on experimental lung injury and fibrosis. Am. J. Respir. Crit. Care Med. 182, 633-642 (2010).

23. Hubner, R. H. et al. Standardized quantification of pulmonary fibrosis in histological samples. BioTechniques 44, 507-511 (2008). 514-507.

24. Burger, R. M., Peisach, J. \& Horwitz, S. B. Activated bleomycin. A transient complex of drug, iron, and oxygen that degrades DNA. J. Biol. Chem. 256, 11636-11644 (1981).

25. van Deursen, J. M. The role of senescent cells in ageing. Nature $\mathbf{5 0 9}, 439-446$ (2014).

26. Aoshiba, K, Tsuji, T. \& Nagai, A. Bleomycin induces cellular senescence in alveolar epithelial cells. Eur. Respir. J. 22, 436-443 (2003).

27. Rayess, H., Wang, M. B. \& Srivatsan, E. S. Cellular senescence and tumor suppressor gene p16. Int. J. Cancer 130, 1715-1725 (2012).

28. Alexander, K., Yang, H. S. \& Hinds, P. W. pRb inactivation in senescent cells leads to an E2F-dependent apoptosis requiring p73. Mol. Cancer Res. 1, 716-728 (2003).

29. Lee, J. M. et al. Involvement of alveolar epithelial cell necroptosis in idiopathic pulmonary fibrosis pathogenesis. Am. J. Respir. Cell Mol. Biol. 59, 215-224 (2018).

30. Tabas, I. \& Ron, D. Integrating the mechanisms of apoptosis induced by endoplasmic reticulum stress. Nat. Cell Biol. 13, 184-190 (2011).

31. Bjoraker, J. A. et al. Prognostic significance of histopathologic subsets in idiopathic pulmonary fibrosis. Am. J. Respir. Crit. Care Med. 157, 199-203 (1998).

32. Araya, J. et al. Insufficient autophagy in idiopathic pulmonary fibrosis. Am. J. Physiol. Lung Cell. Mol. Physiol. 304, L56-L69 (2013).

33. Patel, A. S. et al. Autophagy in idiopathic pulmonary fibrosis. PloS ONE 7, e41394 (2012).

34. Kabeya, Y. et al. LC3, a mammalian homologue of yeast Apg8p, is localized in autophagosome membranes after processing. EMBO J. 19, 5720-5728 (2000).

35. Codogno, P., Mehrpour, M. \& Proikas-Cezanne, T. Canonical and non-canonical autophagy: variations on a common theme of self-eating? Nature reviews. Nat. Rev. Mol. Cell Biol. 13, 7-12 (2011).

36. Waters, D. W. et al. Fibroblast senescence in the pathology of idiopathic pulmonary fibrosis. Am. J. Physiol. Lung Cell. Mol. Physiol. 315, L162-1172 (2018). 
37. Alder, J. K. et al. Telomere dysfunction causes alveolar stem cell failure. Proc. Natl Acad. Sci. USA 112, 5099-5104 (2015)

38. Minagawa, S. et al. Accelerated epithelial cell senescence in IPF and the inhibitory role of SIRT6 in TGF-beta-induced senescence of human bronchial epithelial cells. Am. J. Physiol. Lung Cell. Mol. Physiol. 300, L391-L401 (2011).

39. Schafer, M. J. et al. Cellular senescence mediates fibrotic pulmonary disease. Nat. Commun. 8, 14532 (2017).

40. Kaufman, R. J. Stress signaling from the lumen of the endoplasmic reticulum: coordination of gene transcriptional and translational controls. Genes Dev. 13, 1211-1233 (1999).

41. Wu, J. \& Kaufman, R. J. From acute ER stress to physiological roles of the unfolded protein response. Cell Death Differ. 13, 374-384 (2006).

42. Lawson, W. E. et al. Endoplasmic reticulum stress in alveolar epithelial cells is prominent in IPF: association with altered surfactant protein processing and herpesvirus infection. Am. J. Physiol. Lung Cell. Mol. Physiol. 294, L1119-L1126 (2008).

43. Korfei, M. et al. Epithelial endoplasmic reticulum stress and apoptosis in sporadic idiopathic pulmonary fibrosis. Am. J. Respir. Crit. Care Med. 178 838-846 (2008).

44. Zhong, Q. et al. Role of endoplasmic reticulum stress in epithelialmesenchymal transition of alveolar epithelial cells: effects of misfolded surfactant protein. Am. J. Respir. Cell Mol. Biol. 45, 498-509 (2011).

45. Baek, H. A. et al. Involvement of endoplasmic reticulum stress in myofibroblastic differentiation of lung fibroblasts. Am. J. Respir. Cell Mol. Biol. 46, 731-739 (2012).

46. Rhee, H. J., Kim, E. J. \& Lee, J. K. Physiological polyamines: simple primordial stress molecules. J. Cell. Mol. Med. 11, 685-703 (2007).

47. Wei, C. et al. Spermine inhibits endoplasmic reticulum stress-induced apoptosis: a new strategy to prevent cardiomyocyte apoptosis. Cell. Physiol. Biochem. 38, 531-544 (2016).
48. Seiler, N. \& Raul, F. Polyamines and apoptosis. J. Cell. Mol. Med. 9, 623-642 (2005).

49. Mandal, S., Mandal, A. \& Park, M. H. Depletion of the polyamines spermidine and spermine by overexpression of spermidine/spermine N(1)-acetyltransferase 1 (SAT1) leads to mitochondria-mediated apoptosis in mammalian cells. Biochem. J. 468, 435-447 (2015).

50. Spotheim-Maurizot, M., Ruiz, S., Sabattier, R. \& Charlier, M. Radioprotection of DNA by polyamines. Int. J. Radiat. Biol. 68, 571-577 (1995).

51. $\mathrm{Ha}, \mathrm{H}$. C. et al. The natural polyamine spermine functions directly as a free radical scavenger. Proc. Natl Acad. Sci. USA 95, 11140-11145 (1998).

52. Agostinelli, E. et al. The biological functions of polyamine oxidation products by amine oxidases: perspectives of clinical applications. Amino Acids 27, 347-358 (2004)

53. Qin, L., Wang, Z., Tao, L. \& Wang, Y. ER stress negatively regulates AKT/TSC/ mTOR pathway to enhance autophagy. Autophagy 6, 239-247 (2010).

54. Levine, B. \& Kroemer, G. Autophagy in the pathogenesis of disease. Cell 132 27-42 (2008).

55. Madeo, F., Bauer, M. A., Carmona-Gutierrez, D. \& Kroemer, G. Spermidine: a physiological autophagy inducer acting as an anti-aging vitamin in humans? Autophagy 15, 165-168 (2019).

56. Codogno, P. \& Meijer, A. J. Autophagy and signaling: their role in cell survival and cell death. Cell Death Differ. 12, 1509-1518 (2005).

57. Pegg, A. E. Functions of polyamines in mammals. J. Biol. Chem. 291 14904-14912 (2016)

58. Coleman, C. S., Hu, G. \& Pegg, A. E. Putrescine biosynthesis in mammalian tissues. Biochem. J. 379, 849-855 (2004).

59. North, M. L. et al. Increased ornithine-derived polyamines cause airway hyperresponsiveness in a mouse model of asthma. Am. J. Respir. Cell Mol. Biol. 48, 694-702 (2013)

60. Zhao, Y. D. et al. Metabolic heterogeneity of idiopathic pulmonary fibrosis: a metabolomic study. BMJ Open Respir. Res. 4, e000183 (2017). 